\title{
Targeted metabolomics study of serum bile acid profile in patients with end-stage renal disease undergoing hemodialysis
}

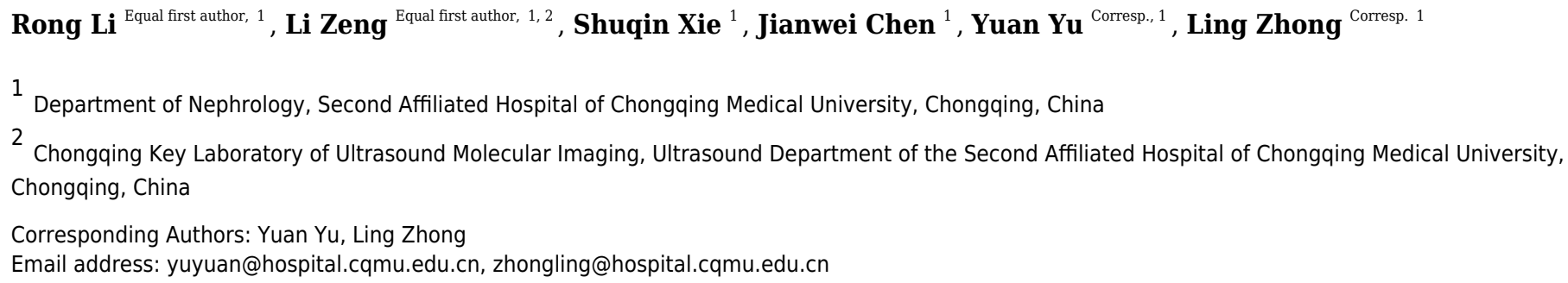

Background. Bile acids are important metabolites of intestinal microbiota, which have profound effects on host health. However, whether metabolism of bile acids is involved in the metabolic complications of end-stage renal disease (ESRD), and the effects of bile acids on the prognosis of ESRD remain obscure. Therefore, this study investigated the relationship between altered bile acid profile and the prognosis of ESRD patients.

Methods. A targeted metabolomics approach based on ultra performance liquid chromatography-tandem mass spectrometry (UPLC-MS/MS) was used to determine the changes in serum bile acids between ESRD patients $(n=77)$ and healthy controls $(n=30)$. Univariate and multivariate statistical analyses were performed to screen the differential proportions of bile acids between the two groups. Results. A total of 6 differentially expressed bile acids were identified as potential biomarkers for differentiating ESRD patients from healthy subjects. The decreased concentrations of chenodeoxycholic acid (CDCA), deoxycholic acid (DCA) and cholic acid (CA) were significantly associated with dyslipidemia in ESRD patients. Subgroup analyses revealed that the significantly increased concentrations of taurocholic acid (TCA), taurochenodeoxycholic acid (TCDCA), taurohyocholic acid (THCA) and tauro $\alpha$-muricholic acid (T $\alpha$ MCA) were correlated to the poor prognosis of ESRD patients. Conclusions. The serum bile acid profile of ESRD patients differed significantly from that of healthy controls. In addition, the altered serum bile acid profile might contribute to the poor prognosis and metabolic complications of ESRD patients. 
1 Targeted metabolomics study of serum bile acid profile in patients with end-stage renal

2 disease undergoing hemodialysis

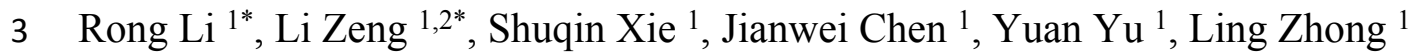

41 Department of Nephrology, Second Affiliated Hospital of Chongqing Medical University, 5 Chongqing, China

$6{ }^{2}$ Chongqing Key Laboratory of Ultrasound Molecular Imaging, Ultrasound Department of the

7 Second Affiliated Hospital of Chongqing Medical University, Chongqing, China

$8{ }^{*}$ The authors contributed equally to this study.

9 Corresponding author:

10 Professor Ling Zhong

11 Department of Nephrology

12 Second Affiliated Hospital of Chongqing Medical University

13 Lingjiang Road, Yuzhong District, Chongqing 400010, China

14 Tel: $(+86) 2363713366$

15 Fax: $(+86) 2363713366$

16 E-mail:zhongling@hospital.cqmu.edu.cn

17 Professor Yuan $\mathrm{Yu}$

18 Department of Nephrology

19 Second Affiliated Hospital of Chongqing Medical University

20 Lingjiang Road, Yuzhong District, Chongqing 400010, China

21 Tel: (+86) 2363713366

22 Fax: $(+86) 2363713366$

23 E-mail: yuyuan@hospital.cqmu.edu.cn 


\section{Abstract}

Background. Bile acids are important metabolites of intestinal microbiota, which have profound effects on host health. However, whether metabolism of bile acids is involved in the metabolic complications of end-stage renal disease (ESRD), and the effects of bile acids on the prognosis of ESRD remain obscure. Therefore, this study investigated the relationship between altered bile acid profile and the prognosis of ESRD patients.

Methods. A targeted metabolomics approach based on ultra performance liquid chromatographytandem mass spectrometry (UPLC-MS/MS) was used to determine the changes in serum bile acids between ESRD patients ( $\mathrm{n}=77)$ and healthy controls $(\mathrm{n}=30)$. Univariate and multivariate statistical analyses were performed to screen the differential proportions of bile acids between the two groups.

Results. A total of 6 differentially expressed bile acids were identified as potential biomarkers for differentiating ESRD patients from healthy subjects. The decreased concentrations of chenodeoxycholic acid (CDCA), deoxycholic acid (DCA) and cholic acid (CA) were significantly associated with dyslipidemia in ESRD patients. Subgroup analyses revealed that the significantly increased concentrations of taurocholic acid (TCA), taurochenodeoxycholic acid (TCDCA), taurohyocholic acid (THCA) and tauro $\alpha$-muricholic acid (TaMCA) were correlated to the poor prognosis of ESRD patients.

Conclusions. The serum bile acid profile of ESRD patients differed significantly from that of healthy controls. In addition, the altered serum bile acid profile might contribute to the poor prognosis and metabolic complications of ESRD patients. 


\section{Introduction}

46 Chronic kidney disease is an emerging health problem in China, with an overall prevalence of

$4710.8 \%$. End-stage renal disease (ESRD) accounts for $0.03 \%$ of the Chinese population, and the total number of ESRD patients has reached approximately 3.3 million (Yano et al. 2012). Loss of renal function in ESRD patients can result in the abnormal metabolism of proteins, fatty acids, carbohydrates, vitamins, water, electrolytes and acid-base, leading to dysfunction of various organ systems and poor quality of life. In recent years, the concept of intestinal-renal axis has been increasingly accepted (Evenepoel et al. 2017), which refers to the interaction between chronic kidney disease and endogenous intestinal microbiota as well as their metabolites that may affect the pathophysiological state of ESRD patients. On the one hand, uremia affects the composition and metabolism of intestinal microbiota (Vaziri et al. 2013; Wang et al. 2012; Wong et al. 2014); on the other hand, disordered intestinal microbiota increases the production of enterogenous urotoxin and impairs the barrier function of intestinal mucosa, thereby aggravating renal microinflammation and dyslipidemia, promoting the progression of chronic kidney disease, and elevating the risks of atherosclerosis, cardiovascular and cerebrovascular diseases (Evenepoel et al. 2017; Vaziri et al. 2016). Hence, enterogenous metabolites have become an important research topic for combating the metabolic complications of chronic kidney disease.

Bile acids are essential metabolites of intestinal microbiota. Primary bile acids are initially synthesized from cholesterol in the liver (Javitt 1994), and then conjugated with taurine or glycine to form conjugated bile acids (Russell 2003), which are secreted into the intestine via the bile duct. These bile acids are mostly reabsorbed at the end of the ileum, and re-transferred through the portal 
66

67

68

69

vein back to the liver, thereby forming a complete enterohepatic circulation of bile acids. The remaining small amount of unreabsorbed bile acids may undergo a series of biotransformation under the action of intestinal microbiota, such as deconjugation, dehydroxylation, dehydrogenation and epimerization, to produce secondary and tertiary bile acids (Midtvedt 1974).

Bile acids have been known to play a pivotal role in promoting the absorption of lipids and fatsoluble nutrients in intestinal tract. More recently, they have also been found to affect lipid, glucose and energy homeostasis by acting as endogenous signaling molecules conjugated to bile acid receptors, including farnesoid X receptor (FXR) and G protein-coupled bile acid receptor (GPBAR) (Hylemon et al. 2009).

At present, ESRD is commonly diagnosed by glomerular filtration rate test $\left(\leq 15 \mathrm{ml} / \mathrm{min} / 1.73 \mathrm{~m}^{2}\right)$. Considering the recent advancements in various "omics" fields such as genomics, epigenomics, transcriptomics, proteomics and metabolomics, the introduction of novel omics techniques may allow for the identification of novel biomarkers in ESRD. Several studies have suggested the potential role of bile acids in chronic kidney disease (Marečková et al. 1990; Jimenez et al. 2002; Chu et al. 2015). However, whether bile acid metabolism is involved in the metabolic disorders of ESRD and the impacts of bile acids on the prognosis of ESRD are still obscure. Thus, the purpose of this study was to investigate the relationship between altered bile acid profile and the prognosis of ESRD patients receiving hemodialysis. UPLC-MS/MS, a well-adopted technique in metabolomics research, was used to quantitatively analyze the bile acid profile of ESRD patients on regular hemodialysis. 


\section{Subjects and methods}

\subsection{Patient recruitment and sample collection}

A total of 77 ESRD patients (43 males and 34 females) who underwent regular hemodialysis at the Department of Nephrology, the Second Affiliated Hospital of Chongqing Medical University from December 2013 to December 2016 were recruited, and followed up for a long period.

Patients were eligible for this study if they were aged between 40 and 70 years, had no previous infections, received no antibiotic treatment within the past three months, had glomerular filtration rate $<15 \mathrm{~mL} / \mathrm{min} / 1.73 \mathrm{~m}^{2}$ (calculated by the Cockcroft-Gault formula), and underwent regular hemodialysis 2-3 times per week. Exclusion criteria were cholestasis or abnormal markers (serum or imaging) for liver function, history of viral hepatitis, alcoholic liver disease or steatohepatitis that may cause liver damage, history of gallbladder disease or gallbladder removal, and concomitant active malignant tumor.

Meanwhile, 30 age- and gender-matched healthy subjects (17 males and 13 females) were selected as a control group from the physical examination center of the Second Affiliated Hospital of Chongqing Medical University. Fasting blood samples were collected from all subjects, and transported on ice to the laboratory within 2 hours of collection. Subsequently, the blood components were separated, aliquoted and stored at $-80^{\circ} \mathrm{C}$ until further analyses. Patient data such as age, gender, height, and weight were collected, and the body mass index (BMI) was calculated as follows: [(weight $(\mathrm{kg}) /$ height $\left.^{2}\left(\mathrm{~m}^{2}\right)\right]$. The present study was approved by the Ethics Committee of the Second Affiliated Hospital of Chongqing Medical University,the approval number was (2018)028. Written informed consent was obtained from all subjects prior to enrollment. 
107

108

109

110

111

112

113

114

115

116

117

118

119

120

121

122

123

124

125

126

127

\subsection{Materials and Reagents}

HPLC-MS grade methanol was purchased from Fisher Scientific. Both formic acid (HPLC grade) and ammonium acetate (HPLC-MS grade) were purchased from Sigma-Aldrich. The authentic standards of both unconjugated and conjugated bile acids were purchased from Steraloids (Newport, RI, USA). Four deuterium-labeled bile acids (CA-d4, CDCA-d4, LCA-d4 and GCAd4) were purchased from Steraloids (Newport, RI, USA) and other six deuterium-labeled bile acids (DCA-d4, GCDCA-d4, GDCA-d4, UDCA-d4, GLCA-d4 and GUDCA-d4) were purchased from Cambridge Isotope Laboratories (Tewksbury, MA, USA). The ten deuterium-labeled bile acids were used as isotope-labeled internal standards (ISs) for quantitation and identification of compounds. Other materials were purchased from Anpel Laboratory Technologies (Shanghai, China).

\subsection{Preparation of standard curves}

Aliquots of each BA standard solution were mixed to generate a stock solution at $1000 \mathrm{ng} / \mathrm{mL}$ in methanol. The stock solution was serially diluted with methanol to 400, 200, 100, 40, 20, 10, 4 and $2 \mathrm{ng} / \mathrm{mL}$. The stock solutions of the 10 ISs were prepared with methanol, and further diluted to a concentration of $100 \mathrm{ng} / \mathrm{mL}$ using methanol. To prepare a standard curve, the above 9 stock solutions were isometrically mixed with $100 \mathrm{ng} / \mathrm{mL}$ of IS solution to generate calibration points covering a concentration range of $1-500 \mathrm{ng} / \mathrm{mL}$ for all the analytes.

\subsection{Serum samples preparation}

A $50-\mu \mathrm{L}$ aliquot of serum sample was mixed with $200 \mu \mathrm{L}$ methanol/acetonitrile solution $(5: 3, \mathrm{v} / \mathrm{v})$.

After centrifugation for $15 \mathrm{~min}$ at $14000 \mathrm{~g}$ and $4{ }^{\circ} \mathrm{C}, 200 \mu \mathrm{L}$ supernatant was dried under a stream 
128 of nitrogen. The residues were re-dissolved in $100 \mu \mathrm{L}$ of $50 \%$ aqueous methanol containing 50

$129 \mathrm{ng} / \mathrm{mL}$ ISs prior to UPLC-MS/MS analysis, with an injection volume of $7.5 \mu \mathrm{L}$.

\section{$130 \quad 2.5$ UPLC-MS/MS analysis}

131 The samples were analyzed by UPLC-MS/MS using an Acquity UPLC system (Waters

132 Corporation, Milford, MA) coupled with a Triple Quad 5500 tandem mass spectrometer (AB

133 Sciex, Framingham, MA). All the samples were injected onto a Waters UPLC BEH C18 column

$134(100 \mathrm{~mm} \times 2.1 \mathrm{~mm}, 1.7 \mu \mathrm{m})$ at a flow rate of $0.35 \mathrm{~mL} / \mathrm{min}$. The mobile phase consisted of $10 \mathrm{mM}$

135 ammonium acetate, $0.012 \%$ formic acid in water (A) and methanol (B). The chromatographic

136 separation was conducted by a gradient elution program as follows: $0-0.5 \mathrm{~min}, 35 \% \mathrm{~B} ; 1 \mathrm{~min}$,

$13760 \% \mathrm{~B} ; 4 \mathrm{~min}, 80 \% \mathrm{~B} ; 5.3-6 \mathrm{~min}, 100 \% \mathrm{~B}$; and $6.1-8 \mathrm{~min}, 35 \% \mathrm{~B}$. The column temperature was set 138 to $45^{\circ} \mathrm{C}$.

139 The analytes eluted from column were ionized in a negative ion mode electrospray ionization (ESI-

140 ) mode. The source temperature was set at $550^{\circ} \mathrm{C}$. The pressures of curtain gas (CUR), ion source 141 gas 1 (GS1), ion source gas 2 (GS2) and collision gas (CAD) were 30, 50, 50 and 8 psi, 142 respectively. The voltage of ion spray (IS) was $-4500 \mathrm{~V}$, while $-10 \mathrm{~V}$ for both entrance potential 143 (EP) and collision cell exit potential (CXP1). Multiple reaction monitoring (MRM) was used to 144 acquire data under optimal conditions of MRM transition (precursor > product), declustering 145 potential (DP) and collision energy (CE), as described in Supplementary Table 1. The optimal 146 dwell time was 8 ms. Both samples and standard curves samples were analyzed simultaneously. 147 Analyst software (version 1.5.2; AB Sciex) was used for instrument control and data acquisition. 
148

149

150

151

152

153

154

155

156

157

158

159

160

161

162

163

164

165

166

167

168

\subsection{Mass spectrometry data pretreatment}

Mass spectrometry data were also analyzed by Analyst version 1.5.2 software (AB Sciex).

The default parameters and assisting manual inspection were adopted to ensure the qualitative and quantitative accuracies of each compound. The peak areas of target compounds were integrated and output for quantitative calculation. Considering the extensive individual variations of BA concentrations, the relative proportion of TBA was calculated for comparison. TBA was defined as a sum of all the 26 BAs associated with ESRD risk. The proportion of each BA was calculated as follow: concentrations of each BA / TBA *100\% (Yu et al. 2015).

\subsection{Statistical analysis}

Statistical analyses were performed using SPSS 24.0 software (SPSS, Chicago, IL, USA). Continuous variables were tested for normal distribution via the Shapiro-Wilk test. Mann-Whitney U test or independent sample t test was used to compare the metabolomic data between ESRD and HC groups. One-way analysis of variance (ANOVA) followed by post-hoc testing was used to compare the metabolomic data among ESRD death group, ESRD survival group and HC group. Least significant difference (LSD) and Dunnett's T3 methods were applied to the absence and presence of heterogeneity of variance, respectively. $\mathrm{P}$ value of less than 0.05 was considered statistically significant.

Multivariate analyses were performed with SIMCA-P+ 14.0 software (Umetrics AB). Principal component analysis (PCA) was used for visual clustering and grouping, as well as initial evaluation of the model's validity. Supervised orthogonal partial least squares-discriminant analysis (OPLSDA) was used to further compare the differences between the two groups, where the effects of 
169

170

171

172

173

174

175

176

177

178

179

180

181

182

183

184

185

186

187

188

189

model fitting were evaluated with R2X, R2Y and Q2Y model parameters. R2X and R2Y represent

the fractions of the variance in $\mathrm{X}$ and $\mathrm{Y}$, respectively, that are explained by OPLS-DA model,

while Q2Y indicates the predictive performance of the model. Permutation test was used to verify

the model in order to avoid over-fitting. Loading plots and variable importance of projection (VIP)

$>1$ were adopted as the criteria for screening differentially expressed bile acids. Next, bile acid

components contributed most to the intergroup difference were identified using univariate

statistical tests (Kondo et al. 2014; Zhu et al. 2015). Receiver operating characteristic (ROC) curve

was plotted to calculate the area under the curve (AUC) in order to assess the diagnostic efficacies

of the differential metabolites.

\section{Results}

\subsection{Demographic and clinical characteristics of patients}

Demographic and clinical characteristics of 107 subjects are shown in Table 1. There were no significant differences between ESRD group and HC group in terms of age, BMI and sex ratio.

With regard to laboratory indices, the levels of serum creatinine (CREA), blood urea nitrogen (BUN), uric acid (UA), phosphorus (P), triglyceride (TG) and total bile acid (TBA) were significantly elevated $(\mathrm{P}<0.05)$ in ESRD patients compared to $\mathrm{HC}$ group, while the levels of blood calcium (Ca), albumin (ALB), total cholesterol (CHOL), high-density lipoprotein cholesterol (HDL-C), low-density lipoprotein cholesterol (LDL-C), hemoglobin (HGB) and platelet count (PLT) were significantly decreased $(\mathrm{P}<0.05)$. These findings were consistent with the general clinical characteristics of ESRD patients. Nonetheless, there were no significant differences in the levels of alanine aminotransferase (ALT), aspartate aminotransferase (AST), alkaline phosphatase 
190 (ALP), total bilirubin (TBIL) and direct bilirubin (DBIL) between the two groups. Subgroup 191 analyses revelaed that ESRD death group had increased mean age, reduced serum creatinine level, 192 and elevated levels of aspartate aminotransferase and total bile acid compared to ESRD survival 193 group. However, no significant differences was found in other parameters between the two 194 subgroups (Table 2).

\subsection{Univariate analyses of serum bile acid profile}

The serum bile acid profiles of 77 ESRD patients and $30 \mathrm{HC}$ were analyzed by HPLC-MS/MS.

The differences in bile acid profile between ESRD group and HC group was compared by Mann-

Whitney $U$ test, and the results are shown in Table 3. A total of 32 bile acid components in serum were quantified, of which 26 bile acids were significantly correlated with ESRD. The proportions of unconjugated bile acids CA, CDCA, DCA, HDCA, UDCA, $\alpha+\omega \mathrm{MCA}, \gamma \mathrm{MCA}, 7 \mathrm{KLCA}$, 12KLCA and 6,7-diketoLCA were all decreased, while only the proportion of unconjugated $\beta M C A$ was significantly increased $(\mathrm{P}<0.05)$ in ESRD group compared to $\mathrm{HC}$ group. The proportion of LCA showed a downward trend, but the difference was not statistically significant $(\mathrm{P}=0.062)$. Altogether, these results indicate that the proportions of most unconjugated bile acids are reduced in ESRD patients. In addition, the proportions of conjugated bile acids GCA, GCDCA, TCA, TCDCA, THCA, TaMCA and TUDCA in ESRD group were significantly higher (all $\mathrm{P}<0.05)$ than those in $\mathrm{HC}$ group. Nevertheless, the proportions of the remaining conjugated bile acids GDCA, GHCA, GLCA, GUDCA, TDCA and T $\beta$ MCA were not significantly different between the two groups. 
211 according to their chemical properties and subsequently compared. The results showed that ESRD

212 patients exhibited lower proportions of total unconjugated bile acids (UnconBA) and secondary

213 bile acids (SBA), while higher proportions of total conjugated bile acids (ConBA), primary bile

214 acids (PBA), taurine-conjugated bile acids (TaurineBA) and glycine-conjugated bile acids

215 (GlycineBA) compared to HC group $(\mathrm{P}<0.05)$.

216

3.3 Multivariate analyses of serum bile acid profile

217 The distribution of bile acids in the samples was preliminarily investigated by PCA in order to

218 verify the rationality of experimental design and the homogeneity of biological replicates. The

219 results of PCA are presented as a three-dimensional score plot (Fig. 1A), where each point

220 represents a serum sample. Notably, a high differentiation was observed between ESRD group and

221 HC group $(\mathrm{R} 2 \mathrm{X}=0.645, \mathrm{Q} 2=0.308)$, with a partial overlap and the top four principal components

222 accounted for $64.5 \%$ of the total variance.

223 OPLS-DA model exhibited higher ability for intergroup comparisons than PCA model. Thus,

224 OPLS-DA scores were plotted to further analyze the difference between ESRD group and HC group, and to identify the variables contributed most to the difference between the two groups. As

shown in Fig. 1B, the serum bile acid profiles of the two groups displayed an obvious separation

trend in OPLS-DA model, with the model parameter values of R2Xcum $=0.564$, R2Ycum $=0.508$

and Q2Ycum $=0.303$. These data indicate that the bile acid metabolic profiles of the two groups

may manifest as different phenotypes.

The results of the permutation test used for validating the OPLS-DA model are presented in Fig.

1C. R2 denotes the explanation capacity of the model, while Q2 represents the predictive capacity 
232 of the model. The low values of intercepts, R2 (0.109) and Q2 (-0.198), revealed that the

233 constructed model was not over-fitting.

234 The most significant variables were visually displayed by a loading plot. The variables with

235 statistical significance in univariate analysis $(\mathrm{P}<0.05)$ and VIP $>1$ were used as the criteria for the

236 identification of differentially expressed bile acids. A total of 6 bile acids (GCDCA, CDCA, DCA,

237 TCDCA, GCA and CA) were identified as potential biomarkers for differentiating ESRD patients

238 from healthy subjects (Table 4).

$239 \quad 3.4$ ROC curve analysis of differentially expressed bile acids

240 The 6 differentially expressed bile acids and TBA screened by OPLS-DA model were presented

241 graphically as ROC curves in order to explore their diagnostic abilities. As shown in Fig. 2, the

242 differentiating ability of TBA $(\mathrm{AUC}=0.701,95 \% \mathrm{CI}: 0.591,0.810 ; \mathrm{P}=0.001)$ was lower than that

243 of the 6 identified bile acids (except for CA). Among the 6 bile acids, CDCA (AUC $=0.815,95 \%$

244 CI: 0.726, 0.904; $\mathrm{P}<0.001$ ) exhibited the highest ability to differentiate ESRD patients from

245 healthy subjects. In addition, a combination of all 6 differential bile acids demonstrated the most

246 distinguishing ability $(\mathrm{AUC}=0.890,95 \% \mathrm{CI}: 0.818,0.963 ; \mathrm{P}<0.001)$, hence can be used as a

247 combined biomarker for ESRD diagnosis.

248 3.5 Analysis of bile acid compositions among the subgroups

249 To further assess the differences in bile acids among ESRD patients with different prognoses, we

250 retrospectively analyzed the survival rates of 47 patients whose samples were collected during

251 2013, and found that 17 patients died over a period of 3 years. Therefore, we established two

252 subgroups consisted of 17 dead patients and 30 survivors, and then compared with HC group. The 
253 results showed that the abundances of TCA, TCDCA, THCA, TaMCA, TaurineBA and TBA in

254 the control group, survival group and death group increased successively (Fig. 3), with a 255 statistically significant difference among the two groups $(\mathrm{P}<0.1)$. Altogether, these findings

256 indicate that poor prognosis and mortality are positively correlated to the concentrations of the

257 above-mentioned bile acids.

\section{Discussion}

259 In this study, we quantitatively analyzed the serum bile acid profiles in ESRD patients and HC using UPLC-MS/MS in order to determine the roles of bile acids in ESRD. The results showed that ESRD patients and HC displayed significant alterations in the proportions of bile acids.

Notably, the levels of TBA were significantly increased in ESRD patients, as consistent with the findings of previous studies (Balestri \& Cupisti 1996). Previous studies have shown that the increased TBA level in ESRD patients is probably caused by subclinical liver damage, abnormal enterohepatic circulation (Jimenez et al. 2002), abnormal reabsorption and secretion of bile acids

by renal tubule, and diminished excretion of bile acids from kidney due to low glomerular filtration rate (Chu et al. 2015). In addition, Gai et al. (2014) suggest that the elevated plasma BA level represents an early event in the progression of chronic renal failure, and is due to increased efflux across the basolateral membrane of human hepatocytes.

The roles of bile acids in the body metabolism and disease onset are difficult to understand by the sheer investigative effort involved in the altering levels of TBA due to their complex bile acids metabolism and diverse chemical structures. Therefore, we classified each bile acid according to its chemical property for the purpose of comparison, and found that ESRD patients exhibited 
274

275

276

277

278

279

decreased proportions of unconjugated bile acids and secondary bile acids, as well as increased proportions of conjugated bile acids and primary bile acids. Increasing evidence has shown that intestinal microbiota regulates the conversion of primary bile acids to secondary bile acids, which in turn affects the size and composition of bile acid pool (Duboc et al. 2013). After the hepatic primary bile acids enter the intestinal tract, the hydrolysis of the amino bond at the 24th carbon atom of bile acid conjugates is catalyzed by bile salt hydrolase (produced by intestinal microbiota) to form unconjugated bile acids. This reaction is followed by $7 \alpha$ dehydroxylation to form secondary bile acids. However, only the free bile acid salts can carry out $7 \alpha$ dehydroxylation. Hence, the conversion of conjugated bile acids to free bile acids by bile salt hydrolase is the premise of $7 \alpha$ dehydroxylation (Begley et al. 2005). Our results suggest that the decreased proportions of unconjugated bile acids and increased proportions of conjugated bile acids in ESRD patients may be related to the low activity of bile salt hydrolase resulted from the disordered intestinal microbiota and diminished hydrolysis of bile acids in the intestinal tract. This may lead to an increase in the synthesis of primary bile acids and a decrease in the synthesis of secondary bile acids.

To further analyze the bile acid compositions that play key roles in the onset and progression of ESRD, 6 typical differential bile acids that differentiate ESRD patients from HCs were screened and identified by OPLS-DA model, including CDCA, DCA, CA, GCDCA, TCDCA and GCA. ROC analysis revealed that the combination of the above 6 bile acids was superior to total or individual bile acids for detecting metabolic complications in ESRD patients. Notably, these 6 bile acids are mainly responsible for the abnormal changes in bile acid metabolism in ESRD patients. 
Bile acids have been well known to act as emulsifiers to promote the absorption of lipids and fat-

296

297

298

299

300

301

302

303

304

305

306

307

308

309

310

311

312

313

314

315

soluble vitamins in the small intestine, but the knowledge of bile acids has been constantly

evolving over the last 20 years. They were recently found to act as endogenous signaling molecules in mammals for activating FXR, GPBAR and cell signaling pathways in vivo. Activation of these receptors and cell signaling pathways is involved in the regulation of many important life processes, including the synthesis, metabolism, and transport of bile acids (Ananthanarayanan et al. 2001; Goodwin et al. 2000; Pircher et al. 2003), blood glucose (Katsuma et al. 2005; Stayrook et al. 2005), and lipoprotein (Hirokane et al. 2004; Watanabe et al. 2004), as well as energy metabolism (Watanabe et al. 2006).

FXR is the most important bile acid receptor, not only expressed in the enterohepatic circulation, but also in the heart and kidneys (Forman et al. 1995; Kawamata et al. 2003). Unconjugated bile acids such as CDCA, DCA, LCA and CA are a group of FXR receptor agonists with high affinity, among which CDCA possesses the highest (Jia et al. 2017; Makishima et al. 1999; Song et al. 2015; Vaquero et al. 2013). FXR activation was shown to reduce circulating triglyceride levels by lowering the production of very low-density lipoprotein and increasing its clearance (Watanabe et al. 2004). Moreover, the serum levels of triglyceride and high-density lipoprotein cholesterol were increased in FXR-deficient mice (Lefebvre et al. 2009). The enterohepatic circulation of bile acids is disturbed in hyperlipidemia patients receiving bile acid sequestrants or patients undergoing ileectomy, thus leading to an increase in plasma triglyceride level (Angelin et al. 1978; Buchwald et al. 1990). Interestingly, oral administration of CDCA contributes to a significantly greater reduction in triglycerides compared to placebo (Carulli et al. 2013). Likewise, our study found that 
316 the levels of CDCA, DCA and CA were decreased in ESRD patients, suggesting that the inhibition

317 of FXR activity may increase plasma triglyceride levels, which represents one of the mechanisms

318 of dyslipidemia in ESRD patients.

319 In addition, our study found that the serum levels of cholesterol and low-density lipoprotein

320 cholesterol decreased remarkably in ESRD patients (Table 1). The serum cholesterol levels in

321 patients with chronic renal failure and patients undergoing hemodialysis may be different from

322 those in healthy population. The lower the serum cholesterol level $(50-150 \mathrm{mg} / \mathrm{dl})$, the higher the

323 mortality rate, so-called a "reverse epidemiology" phenomenon (Kalantarzadeh et al. 2003). The

324 activation of FXR and its related signaling pathways has been found to reduce bile acid synthesis

325 by decreasing the biological activity of rate-limiting enzyme CYP7A1, and leading to an increase

326 in serum cholesterol level (Lefebvre et al. 2009). It is speculated that the FXR activity in ESRD

327 patients can be inhibited due to the decreased expression of FXR agonists, which may explain the

328 lower levels of serum cholesterol and low-density lipoprotein cholesterol in ESRD patients

329 compared to HC.

330 Subgroup analysis was performed on ESRD patients with differing prognoses and HC, and the

331 results showed that the abundances of TCA, TCDCA, THCA, TaMCA, TaurineBA and TBA were

332 significantly increased in ESRD death group compared to ESRD survival group (Figure 3).

333 Noticeably, the above bile acids with high abundances were all taurine-conjugated bile acids.

334 These findings demonstrate that the increased taurine-conjugated bile acid levels are positively

335 correlated to the poor prognosis of ESRD patients. Besides, the increased levels of taurine-

336 conjugated bile acids could also promote the absorption of lipids in the small intestine, and increase 
337 the risk of hyperlipidemia and subsequent cardiovascular diseases. It has been reported that free

338 taurine maintains the barrier function of intestinal epithelia by promoting NLRP6 inflammasome

339 activation and enhancing intestinal epithelial IL-18 production (Levy et al. 2015). Therefore, it is

340 speculated that the elevated levels of taurine-conjugated bile acids can reduce the concentrations

341 of free taurine in ESRD patients, leading to an impaired intestinal epithelia barrier function and

342 increased enterogenous urotoxin accumulation.

343 Of the 6 bile acids associated with ESRD risk, only TCDCA was differentially expressed between

344 ESRD death group and ESRD survival group. The other metabolites (i.e. CA, CDCA, DCA, GCA

and GCDCA) were not significantly different between ESRD death and survival groups. These

results indicate that these metabolic biomarkers have no role in the prognosis of ESRD. It is

347 postulated that abnormal bile acid metabolism occurs during the early stage of chronic kidney

348 disease, which does not aggravate ESRD progression and prognosis. The significant roles of

349 TCDCA in the diagnosis and prognosis of ESRD patients remain largely unknown, which warrants

350 further studies.

351 There are still some shortcomings in this study. First, the study subjects were selected from a single

352 center, thus the sample size was relatively small. Second, in the subgroup analyses, the average

age of ESRD patients who died during the three-year follow-up was older than that of ESRD

survival group, which may influence the findings of bile acid compositions between the two

subgroups. Third, the mechanism of bile acid profile change affecting the prognosis of ESRD

patients was speculated on the basis of the existing researches. Fourth, the elevated levels of 
358 altered enterohepatic circulation and/or hepatic dysfunction. Thus, further in vitro, in vivo and 359 well-designed clinical studies are warranted to confirm the usefulness of bile acids in ESRD 360 diagnosis and prognosis. Despite these limitations, this is the first study that utilizes metabolomics 361 approach to identify a panel of bile acid biomarkers for the diagnosis and prognosis of ERSD. 362 Given that the currently available diagnostic methods can only examine the symptoms of ESRD, 363 this metabolomics approach is more appropriately used to describe the etiological mechanisms of 364 the disease.

\section{Conclusion}

366 In conclusion, this study reveals that the serum bile acid profile of ESRD patients is significantly 367 different from that of healthy subjects. In addition, the altered serum bile acid profile may affect 368 the occurrence of metabolic complications and prognosis of ESRD patients. Therefore, targeting 369 bile acid compositions may provide a novel therapeutic strategy for the treatment of relevant 370 metabolic complications in ESRD patients. 


\section{Figure Legends}

372 Fig. 1 Metabolomic analysis of serum samples.

373 (A) PCA score plots. Ellipse:Hotelling's T2 (95\%). (B) OPLS-DA score plots showing a clear 374 discrimination between ESRD (blue sphere) and HC subjects (red octahedron). Ellipse:Hotelling's 375 T2 (95\%). (C) Permutation test demonstrating the robustness of OPLS-DA model. R2=0.109 and $376 \quad \mathrm{Q} 2=-0.198$.

377 Fig. 2 ROC curves for bile acid biomarkers.

ROC curves analysis for the predictive power of 6 bile acid biomarkers and TBA for distinguishing

379 ESRD from healthy control.The final logistic model included six bile acid biomarkers. Using a cutoff probability of $50 \%$, we obtained sensitivity of $94.8 \%$ and specificity of $73.3 \%$ by ROC. The calculated area under the ROC curve was 0.890 (95\% confidence intervals, $0.818,0.963)$.

Fig. 3 Differential serum bile acid profiles among the two cohorts.

The fold-changes in bile acid levels between ESRD death and survival groups (relative to the control cohort) are graphically displayed. Normally-distributed data are depicted in a bar graph with means and standard errors of the mean (SEMs). ${ }^{\mathrm{P}}<0.1 ; * * \mathrm{P}<0.05 ; * * * \mathrm{P}<0.01$ 


\section{References}

Ananthanarayanan M, Balasubramanian N, Makishima M, Mangelsdorf DJ, and Suchy FJ. 2001. Human Bile Salt Export Pump Promoter Is Transactivated by the Farnesoid X Receptor/Bile Acid Receptor. Journal of Biological Chemistry 276:28857-28865.

Angelin B, Einarsson K, Hellström K, and Leijd B. 1978. Effects of cholestyramine and chenodeoxycholic acid on the metabolism of endogenous triglyceride in hyperlipoproteinemia. Journal of Lipid Research 19:1017-1024.

Balestri PL, and Cupisti A. 1996. Elevated Bile Acid Serum Concentrations in Uremics. Nephron 72:325-325.

Begley M, Gahan CG, and Hill C. 2005. The interaction between bacteria and bile. FEMS Microbiology Reviews 29:625-651.

Buchwald H, Varco RL, Matts JP, Long JM, Fitch LL, Campbell GS, Pearce MB, Yellin AE, Edmiston WA, and Jr SR. 1990. Effect of partial ileal bypass surgery on mortality and morbidity from coronary heart disease in patients with hypercholesterolemia. Report of the Program on the Surgical Control of the Hyperlipidemias (POSCH). New England Journal of Medicine 323:946-955.

Carulli N, Ponz dLM, Podda M, Zuin M, Strata A, Frigerio G, and Digrisolo A. 2013. Chenodeoxycholic acid and ursodeoxycholic acid effects in endogenous hypertriglyceridemias. A controlled double-blind trial. Journal of Clinical Pharmacology 21:436-442.

Chu L, Zhang K, Zhang Y, Jin X, and Jiang H. 2015. Mechanism underlying an elevated serum bile acid level in chronic renal failure patients. International Urology and Nephrology 47:345-351.

Duboc H, Rajca S, Rainteau D, Benarous D, Maubert MA, Quervain E, Thomas G, Barbu V, Humbert L, and Despras G. 2013. Connecting dysbiosis, bile-acid dysmetabolism and gut inflammation in inflammatory bowel diseases. Gut 62:531-539.

Evenepoel P, Poesen R, and Meijers B. 2017. The gut-kidney axis. Pediatric Nephrology 32:2005-2014.

Forman BM, Goode E, Chen J, Oro AE, Bradley DJ, Perlmann T, Noonan DJ, Burka LT, Mcmorris T, and Lamph WW. 1995. Identification of a nuclear receptor that is activated by farnesol metabolites. Cell 81:687-693.

Gai Z, Chu L, Hiller C, Arsenijevic D, Penno CA, Montani JP, Odermatt A, Kullak-Ublick GA. 2014. Effect of chronic renal failure on the hepatic, intestinal, and renal expression of bile acid transporters. American Journal of Physiology-Renal Physiology 306:F130-F137.

Goodwin B, Jones SA, Price RR, Watson MA, Mckee DD, Moore LB, Galardi C, Wilson JG, Lewis MC, and Roth ME. 2000. A regulatory cascade of the nuclear receptors FXR, SHP-1, and LRH-1 represses bile acid biosynthesis. Molecular Cell 6:517-526.

Hirokane H, Nakahara M, Tachibana S, Shimizu M, and Sato R. 2004. Bile Acid Reduces the Secretion of Very Low Density Lipoprotein by Repressing Microsomal Triglyceride Transfer Protein Gene Expression Mediated by Hepatocyte Nuclear Factor-4. Journal of Biological Chemistry 279:45685-45692.

Hylemon PB, Zhou H, Pandak WM, Ren S, Gil G, and Dent P. 2009. Bile acids as regulatory molecules. Journal of Lipid Research 50:1509-1520.

Javitt NB. 1994. Bile acid synthesis from cholesterol: regulatory and auxiliary pathways. Faseb Journal Official Publication of the Federation of American Societies for Experimental Biology 8:1308-1311.

Jia W, Xie G, and Jia W. 2017. Bile acid-microbiota crosstalk in gastrointestinal inflammation and carcinogenesis. Nature Reviews Gastroenterology \& Hepatology 15:111-128.

Jimenez F, Monte MJ, El-Mir MY, Pascual MJ, and Marin JJ. 2002. Chronic renal failure-induced changes in serum 
and urine bile acid profiles. Digestive Diseases and Sciences 47:2398-2406.

Kalantarzadeh K, Block G, Humphreys MH, and Kopple JD. 2003. Reverse epidemiology of cardiovascular risk factors in maintenance dialysis patients. Kidney International 63:793-808.

Katsuma S, Hirasawa A, and Tsujimoto G. 2005. Bile acids promote glucagon-like peptide-1 secretion through TGR5 in a murine enteroendocrine cell line STC-1. Biochemical and Biophysical Research Communications 329:386-390.

Kawamata Y, Fujii R, Hosoya M, Harada M, Yoshida H, Miwa M, Fukusumi S, Habata Y, Itoh T, and Shintani Y. 2003. A G Protein-coupled Receptor Responsive to Bile Acids. Journal of Biological Chemistry 278:9435-9440.

Kondo E, Marriott PJ, Parker RM, Kouremenos KA, Morrison P, and Adams M. 2014. Metabolic profiling of yeast culture using gas chromatography coupled with orthogonal acceleration accurate mass time-of-flight mass spectrometry: Application to biomarker discovery. Analytica Chimica Acta 807:135-142.

Lefebvre P, Cariou B, Lien F, Kuipers F, and Staels B. 2009. Role of Bile Acids and Bile Acid Receptors in Metabolic Regulation. Physiological Reviews 89:147-191.

Levy M, Thaiss CA, Zeevi D, Dohnalová L, Zilbermanschapira G, Mahdi JA, David E, Savidor A, Korem T, and Herzig Y. 2015. Microbiota-modulated metabolites shape the intestinal microenvironment by regulating NLRP6 inflammasome signaling. Cell 163:1428-1443.

Marečková O, Skala I, Mareček Z, Malý J, Kočandrle V, Schück O and Prat V. 1990. Bile composition in patients with chronic renal insufficiency. Nephrology Dialysis Transplantation 5: 423-425.

Makishima M, Okamoto AY, Repa JJ, Tu H, Learned RM, Luk A, Hull MV, Lustig KD, Mangelsdorf DJ, and Shan B. 1999. Identification of a nuclear receptor for bile acids. Science 284:1362-1365.

Midtvedt T. 1974. Microbial bile acid transformation. American Journal of Clinical Nutrition 27:1341-1347.

Pircher PC, Kitto JL, Petrowski ML, Tangirala RK, Bischoff ED, Schulman IG, and Westin SK. 2003. Farnesoid X receptor regulates bile acid-amino acid conjugation. Journal of Biological Chemistry 278:27703-27711.

Russell DW. 2003. The enzymes, regulation, and genetics of bile acid synthesis. Annual Review of Biochemistry 72:137-174.

Song P, Rockwell CE, Cui JY, and Klaassen CD. 2015. Individual bile acids have differential effects on bile acid signaling in mice. Toxicology and Applied Pharmacology 283:57-64.

Stayrook KR, Bramlett KS, Savkur RS, Ficorilli J, Cook T, Christe ME, Michael LF, and Burris TP. 2005. Regulation of carbohydrate metabolism by the farnesoid X receptor. Endocrinology 146:984-991.

Vaquero J, Monte MJ, Dominguez M, Muntané J, and Marin JJ. 2013. Differential activation of the human farnesoid $\mathrm{X}$ receptor depends on the pattern of expressed isoforms and the bile acid pool composition. Biochemical Pharmacology 86:926-939.

Vaziri ND, Wong J, Pahl M, Piceno YM, Yuan J, Desantis TZ, Ni Z, Nguyen TH, and Andersen GL. 2013. Chronic kidney disease alters intestinal microbial flora. Kidney International 83:308-315.

Vaziri ND, Zhao YY, and Pahl MV. 2016. Altered intestinal microbial flora and impaired epithelial barrier structure and function in CKD: the nature, mechanisms, consequences and potential treatment. Nephrology Dialysis Transplantation 31:737-746.

Wang IK, Lai HC, Yu CJ, Liang CC, Chang CT, Kuo HL, Yang YF, Lin CC, Lin HH, and Liu YL. 2012. Real-time PCR analysis of the intestinal microbiotas in peritoneal dialysis patients. Applied and Environmental Microbiology 78:1107-1112.

Watanabe M, Houten SM, Mataki C, Christoffolete MA, Kim BW, Sato H, Messaddeq N, Harney JW, Ezaki O, and 
467

468

469

470

471

472

473

474

475

476

477

478

479

480

481

482

483

Kodama T. 2006. Bile acids induce energy expenditure by promoting intracellular thyroid hormone activation. Nature 439:484-489.

Watanabe M, Houten SM, Wang L, Moschetta A, Mangelsdorf DJ, Heyman RA, Moore DD, and Auwerx J. 2004. Bile acids lower triglyceride levels via a pathway involving FXR, SHP, and SREBP-1c. Journal of Clinical Investigation 113:1408-1418.

Wong J, Piceno YM, Desantis TZ, PahI M, Andersen GL, and Vaziri ND. 2014. Expansion of Urease- and UricaseContaining, Indole- and p-Cresol-Forming and Contraction of Short-Chain Fatty Acid-Producing Intestinal Microbiota in ESRD. American Journal of Nephrology 39:230-237.

Zhang L, Wang F, Wang L, Wang W, Liu B, Liu J, Chen M, He Q, Liao Y, Yu X, Chen N, Zhang J-e, Hu Z, Liu F, Hong D, Ma L, Liu H, Zhou X, Chen J, Pan L, Chen W, Wang W, Li X, and Wang H. 2012. Prevalence of chronic kidney disease in China: a cross-sectional survey. The Lancet 379:815-822.

Yu H, Ni Y, Bao Y, Zhang P, Zhao A, Chen T, Xie G, Tu Y, Zhang L, and Su M. 2015. Chenodeoxycholic acid as a potential prognostic marker for Roux-en-Y gastric bypass in Chinese obese patients. The Journal of Clinical Endocrinology \& Metabolism 100:4222-4230.

Zhu J, Djukovic D, Deng L, Gu H, Himmati F, Zaid MA, Chiorean EG, and Raftery D. 2015. Targeted serum metabolite profiling and sequential metabolite ratio analysis for colorectal cancer progression monitoring. Analytical and Bioanalytical Chemistry 407:7857-7863. 
Table $\mathbf{1}$ (on next page)

Demographic and clinical characteristics of ESRD and control group 
1 Table 1 Demographic and clinical characteristics of ESRD and control group

\begin{tabular}{|c|c|c|c|}
\hline Variables & $\begin{array}{c}\text { ESRD } \\
(\mathrm{N}=77)\end{array}$ & $\begin{array}{c}\mathrm{HC} \\
(\mathrm{N}=30) \\
\end{array}$ & P-value \\
\hline Age (year) & $59 \pm 13.9$ & $59.3 \pm 6.8$ & 0.956 \\
\hline $\operatorname{Sex}(M / F)$ & $77(43 / 34)$ & $30(17 / 13)$ & 0.939 \\
\hline $\operatorname{BMI}\left(\mathrm{kg} / \mathrm{m}^{2}\right)$ & $23 \pm 3.2$ & $22.1 \pm 1.5$ & 0.091 \\
\hline PTH（pg/ml） & $246.7 \pm 47.9$ & - & - \\
\hline CREA（umol/L） & $905 \pm 268.9^{\mathrm{a}}$ & $65.4 \pm 14.7$ & $<0.001$ \\
\hline BUN $(\mathrm{mmol} / \mathrm{L})$ & $22.6 \pm 6.2^{\mathrm{a}}$ & $5.2 \pm 1.1$ & $<0.001$ \\
\hline UA（umol/L ） & $444.9 \pm 110.8^{\mathrm{a}}$ & $284.3 \pm 66.7$ & $<0.001$ \\
\hline $\mathrm{Ca}(\mathrm{mmol} / \mathrm{L})$ & $2.2 \pm 0.2^{\mathrm{a}}$ & $2.4 \pm 0.2$ & $<0.001$ \\
\hline $\mathrm{P}(\mathrm{mmol} / \mathrm{L})$ & $1.8 \pm 0.5^{\mathrm{a}}$ & $1.0 \pm 0.1$ & $<0.001$ \\
\hline TG $(\mathrm{mmol} / \mathrm{L})$ & $1.9 \pm 1.1^{\mathrm{a}}$ & $1.1 \pm 0.5$ & $<0.001$ \\
\hline $\mathrm{CHOL}(\mathrm{mmol} / \mathrm{L})$ & $3.5 \pm 1.0^{\mathrm{a}}$ & $4.7 \pm 0.5$ & $<0.001$ \\
\hline HDL-C(mmol/L) & $1.0 \pm 0.4^{\mathrm{a}}$ & $1.5 \pm 0.3$ & $<0.001$ \\
\hline LDL-C(mmol/L) & $1.7 \pm 0.7^{\mathrm{a}}$ & $2.8 \pm 0.5$ & $<0.001$ \\
\hline $\mathrm{WBC}\left(10^{\wedge 9} / \mathrm{L}\right)$ & $5.7 \pm 1.7$ & $5.4 \pm 1.3$ & 0.416 \\
\hline $\operatorname{HGB}(\mathrm{g} / \mathrm{L})$ & $106 \pm 17.2^{\mathrm{a}}$ & $140 \pm 19$ & $<0.001$ \\
\hline $\operatorname{PLT}\left(10^{\wedge 9} / \mathrm{L}\right)$ & $151 \pm 62.6^{\mathrm{a}}$ & $193.7 \pm 50.6$ & 0.003 \\
\hline GLU（mmol/L $)$ & $6.1 \pm 2.8$ & $5.4 \pm 0.9$ & 0.061 \\
\hline ALB $(g / L)$ & $38.3 \pm 4.0^{\mathrm{a}}$ & $44.8 \pm 3.0$ & $<0.001$ \\
\hline AST（U/L） & $20 \pm 16.3$ & $18.1 \pm 7.5$ & 0.548 \\
\hline $\operatorname{ALT}(\mathrm{U} / \mathrm{L})$ & $13.4 \pm 8.3$ & $15.7 \pm 6.3$ & 0.190 \\
\hline ALP(U/L) & $96.2 \pm 47.2$ & $92.2 \pm 28.8$ & 0.675 \\
\hline TBIL(umol/L) & $6.9 \pm 2.8$ & $7.3 \pm 2.3$ & 0.459 \\
\hline DBIL(umol/L) & $3.3 \pm 1.5$ & $3.3 \pm 1.9$ & 0.920 \\
\hline TBA(ng/ml) & $3151.4 \pm 270.0^{\mathrm{a}}$ & $1849.2 \pm 269.8$ & 0.001 \\
\hline
\end{tabular}

Data represent means \pm SD or means \pm SEM.Comparisons performed via t-test (ESRD vs HC), ${ }^{\text {a }} \mathrm{P}<0.05$. 
Table 2 (on next page)

Demographic and clinical characteristics of ESRD Survival,ESRD death and control group 
1 Table 2 Demographic and clinical characteristics of ESRD Survival,ESRD death and control group.

\begin{tabular}{|c|c|c|c|}
\hline Variables & $\begin{array}{c}\text { ESRD } \\
\text { Survival }(\mathrm{N}=30)\end{array}$ & $\begin{array}{c}\text { ESRD } \\
\text { death }(\mathrm{N}=17)\end{array}$ & $\begin{array}{c}\mathrm{HC} \\
(\mathrm{N}=30) \\
\end{array}$ \\
\hline Age(year) & $55.9 \pm 13.5^{\mathrm{b}}$ & $70.7 \pm 9.0^{\mathrm{a}}$ & $59.3 \pm 6.8$ \\
\hline $\operatorname{Sex}(M / F)$ & $30(17 / 13)$ & $17(9 / 8)$ & $30(17 / 13)$ \\
\hline Duration(months) & $30 \pm 21$ & $29 \pm 22$ & - \\
\hline $\operatorname{BMI}\left(\mathrm{kg} / \mathrm{m}^{2}\right)$ & $23.8 \pm 3.2$ & $21.5 \pm 3.1$ & $22.1 \pm 1.5$ \\
\hline PTH(pg/ml) & $338.0 \pm 104.1$ & $240.8 \pm 82.7$ & - \\
\hline CREA(umol/L) & $939.3 \pm 301.5^{\mathrm{ab}}$ & $731.1 \pm 206.8^{\mathrm{a}}$ & $65.4 \pm 14.7$ \\
\hline BUN(mmol/L) & $22.2 \pm 5.4^{\mathrm{a}}$ & $21.4 \pm 7.5^{\mathrm{a}}$ & $5.2 \pm 1.1$ \\
\hline UA(umol/L) & $444.0 \pm 99.6^{\mathrm{a}}$ & $442.4 \pm 113.6^{\mathrm{a}}$ & $284.3 \pm 66.7$ \\
\hline $\mathrm{Ca}(\mathrm{mmol} / \mathrm{L})$ & $2.2 \pm 0.2^{\mathrm{a}}$ & $2.2 \pm 0.3^{\mathrm{a}}$ & $2.4 \pm 0.2$ \\
\hline $\mathrm{P}(\mathrm{mmol} / \mathrm{L})$ & $1.7 \pm 0.4^{\mathrm{a}}$ & $1.6 \pm 0.6^{\mathrm{a}}$ & $1.0 \pm 0.1$ \\
\hline $\mathrm{TG}(\mathrm{mmol} / \mathrm{L})$ & $2.2 \pm 1.4^{\mathrm{a}}$ & $2.1 \pm 1.1^{\mathrm{a}}$ & $1.1 \pm 0.5$ \\
\hline $\mathrm{CHOL}(\mathrm{mmol} / \mathrm{L})$ & $3.9 \pm 0.8^{\mathrm{a}}$ & $3.7 \pm 1.0^{\mathrm{a}}$ & $4.7 \pm 0.5$ \\
\hline HDL-C(mmol/L) & $1.0 \pm 0.4^{\mathrm{a}}$ & $1.0 \pm 0.4^{\mathrm{a}}$ & $1.5 \pm 0.3$ \\
\hline LDL-C(mmol/L) & $2.1 \pm 0.7^{\mathrm{a}}$ & $1.7 \pm 0.8^{\mathrm{a}}$ & $2.8 \pm 0.5$ \\
\hline $\mathrm{WBC}\left(10^{\wedge 9} / \mathrm{L}\right)$ & $5.6 \pm 1.4$ & $5.7 \pm 1.8$ & $5.4 \pm 1.3$ \\
\hline $\operatorname{HGB}(\mathrm{g} / \mathrm{L})$ & $112.5 \pm 16.0^{\mathrm{a}}$ & $103.8 \pm 20.8^{a}$ & $140 \pm 19$ \\
\hline $\operatorname{PLT}\left(10^{\wedge 9} / \mathrm{L}\right)$ & $171.6 \pm 67.1$ & $144.2 \pm 72.4^{\mathrm{a}}$ & $193.7 \pm 50.6$ \\
\hline $\mathrm{GLU}(\mathrm{mmol} / \mathrm{L})$ & $6.1 \pm 3.5$ & $6.9 \pm 2.6$ & $5.4 \pm 0.9$ \\
\hline $\operatorname{ALB}(\mathrm{g} / \mathrm{L})$ & $38.9 \pm 4.2^{\mathrm{a}}$ & $38.4 \pm 4.3^{\mathrm{a}}$ & $44.8 \pm 3.0$ \\
\hline AST(U/L) & $15.6 \pm 6.9^{b}$ & $22.4 \pm 11.4$ & $18.1 \pm 7.5$ \\
\hline $\operatorname{ALT}(\mathrm{U} / \mathrm{L})$ & $12.8 \pm 7.1$ & $14.9 \pm 9.5$ & $15.7 \pm 6.3$ \\
\hline ALP(U/L) & $95.1 \pm 49.0$ & $113.1 \pm 50.5$ & $92.2 \pm 28.8$ \\
\hline TBIL(umol/L) & $6.4 \pm 1.8$ & $7.2 \pm 2.3$ & $7.3 \pm 2.3$ \\
\hline DBIL(umol/L) & $3.2 \pm 1.0$ & $3.7 \pm 1.2$ & $3.3 \pm 1.9$ \\
\hline TBA(ng/ml) & $2888.83 \pm 373.4^{\mathrm{ab}}$ & $4213.0 \pm 785.7^{\mathrm{a}}$ & $1849.2 \pm 269.8$ \\
\hline
\end{tabular}

2 Data represent means \pm SD or means \pm SEM.Comparisons performed via one-way ANOVA. ${ }^{a} \mathrm{P}<0.05$ vs. HC cohort; ${ }^{b} \mathrm{P}<0.05$

3 vs.ESRD death cohort. 
Table 3(on next page)

The percentages of individual bile acid relative to TBA in ESRD and heathy control group(\%) 
Table 3. The percentages of individual bile acid relative to TBA in ESRD and heathy control group $(\%)$

\begin{tabular}{|c|c|c|c|c|}
\hline Metabolite & $\begin{array}{c}\text { ESRD } \\
(\mathrm{N}=77)\end{array}$ & $\begin{array}{c}\mathrm{HC} \\
(\mathrm{N}=30)\end{array}$ & Z-value & P-value \\
\hline $\mathrm{CA}$ & $1.32(0.71 \sim 2.82)$ & $2.43(1.06 \sim 6.56)$ & 2.927 & 0.003 \\
\hline CDCA & $3.86(1.88 \sim 8.10)$ & 14.76(6.49 22.74) & 5.049 & $<0.001$ \\
\hline DCA & $1.37(0.21 \sim 4.37)$ & $6.50(1.39 \sim 17.28)$ & 3.544 & $<0.001$ \\
\hline HDCA & $0.13(0.05 \sim 0.38)$ & $0.51(0.08 \sim 1.72)$ & 2.592 & 0.010 \\
\hline LCA & $0.10(0.02 \sim 0.25)$ & $0.26(0.00 \sim 0.64)$ & 1.865 & 0.062 \\
\hline UDCA & $0.48(0.17 \sim 1.06)$ & $1.17(0.71 \sim 1.89)$ & 3.024 & 0.002 \\
\hline$\alpha+\omega \mathrm{MCA}$ & $0.10(0.03 \sim 0.29)$ & $0.39(0.13 \sim 0.93)$ & 2.871 & 0.004 \\
\hline$\beta \mathrm{MCA}$ & $0.05(0.00 \sim 0.17)$ & $0.00(0.00 \sim 0.00)$ & -3.700 & $<0.001$ \\
\hline$\gamma \mathrm{MCA}$ & $0.48(0.15 \sim 1.05)$ & $0.93(0.50 \sim 2.23)$ & 3.329 & 0.001 \\
\hline 7KLCA & $0.22(0.12 \sim 0.48)$ & $0.48(0.28 \sim 0.70)$ & 3.440 & 0.001 \\
\hline $12 \mathrm{KLCA}$ & $0.07(0.00 \sim 0.22)$ & $0.36(0.00 \sim 1.03)$ & 2.646 & 0.008 \\
\hline 6,7-diketoLCA & $0.08(0.00 \sim 0.22)$ & $0.23(0.04 \sim 0.40)$ & 2.330 & 0.020 \\
\hline GCA & $11.06(8.22 \sim 14.88)$ & $6.13(4.04 \sim 8.76)$ & -4.383 & $<0.001$ \\
\hline GCDCA & $41.62(33.90 \sim 48.88)$ & $30.44(25.60 \sim 38.81)$ & -4.016 & $<0.001$ \\
\hline GDCA & $2.59(0.20 \sim 8.16)$ & $5.53(0.97 \sim 11.10)$ & 1.298 & 0.194 \\
\hline GHCA & $0.52(0.32 \sim 0.90)$ & $0.48(0.35 \sim 0.76)$ & -0.194 & 0.846 \\
\hline GLCA & $0.02(0.00 \sim 0.20)$ & $0.09(0.00 \sim 0.26)$ & 0.813 & 0.416 \\
\hline GUDCA & $8.79(4.93 \sim 13.32)$ & $6.67(2.76 \sim 11.00)$ & -1.345 & 0.178 \\
\hline TCA & $2.52(1.66 \sim 3.77)$ & $1.13(0.61 \sim 1.57)$ & -5.229 & $<0.001$ \\
\hline TCDCA & $5.33(3.69 \sim 9.71)$ & $2.56(1.79 \sim 3.88)$ & -4.834 & $<0.001$ \\
\hline TDCA & $0.67(0.09 \sim 1.68)$ & $0.74(0.16 \sim 1.44)$ & 0.097 & 0.922 \\
\hline THCA & $0.13(0.08 \sim 0.26)$ & $0.11(0.06 \sim 0.15)$ & -2.057 & 0.040 \\
\hline $\mathrm{T} \alpha \mathrm{MCA}$ & $0.10(0.06 \sim 0.16)$ & $0.05(0.00 \sim 0.07)$ & -4.483 & $<0.001$ \\
\hline $\mathrm{T} \beta \mathrm{MCA}$ & $0.45(0.27 \sim 0.71)$ & $0.42(0.24 \sim 0.67)$ & -0.388 & 0.698 \\
\hline TUDCA & $0.63(0.36 \sim 1.41)$ & $0.33(0.24 \sim 0.67)$ & -3.593 & $<0.001$ \\
\hline UnconBA & $12.22(6.77 \sim 20.86)$ & $35.65(22.65 \sim 52.91)$ & 6.124 & $<0.001$ \\
\hline TaurineBA & $11.38(8.05 \sim 17.91)$ & $5.78(4.21 \sim 8.42)$ & -4.459 & $<0.001$ \\
\hline GlycineBA & $71.15(65.22 \sim 80.59)$ & $54.54(42.78 \sim 66.70)$ & -4.862 & $<0.001$ \\
\hline ConBA & 87.78(79.14 93.23) & $64.35(47.09 \sim 77.35)$ & -6.124 & $<0.001$ \\
\hline PBA & 74.95(65.37 83.39) & $65.49(54.34 \sim 74.97)$ & -3.274 & 0.001 \\
\hline SBA & $20.43(12.13 \sim 29.42)$ & $26.20(17.70 \sim 36.79)$ & 2.303 & 0.021 \\
\hline $\mathrm{PBA} / \mathrm{SBA}$ & $3.63(2.15 \sim 7.09)$ & $2.65(1.44 \sim 4.40)$ & -2.538 & 0.011 \\
\hline UnconBA/ConBA & $0.14(0.07 \sim 0.26)$ & $0.55(0.29 \sim 1.13)$ & 6.124 & $<0.001$ \\
\hline
\end{tabular}

2 Data presented as median and inter-quartile range (IQR).

3 Abbreviations: CA, cholic acid;CDCA, chenodeoxycholic acid; DCA, deoxycholic acid; HDCA, hyodeoxycholic acid; LCA, 4 lithocholic acid; UDCA, ursodeoxycholic acid; $\alpha+\omega$ MCA, $\alpha$-muricholic acid and $\omega$-muricholic acid; $\beta$ MCA, $\beta$-muricholic acid; $5 \gamma \mathrm{MCA}, \gamma$-murocholic acid; 7KLCA, 7-ketolithocholic acid; 12KLCA, 12-ketolithocholic acid; 6,7-diketoLCA, 6,76 diketolithocholic acid; GCA, glycocholic acid; GCDCA, glycochenodeoxycholic acid;GDCA, glycodeoxycholic acid; GHCA, 
7 glycohyocholic acid; GLCA, glycolithocholic acid; GUDCA, glycoursodeoxycholic acid; TCDCA, taurochenodeoxycholic 8 acid;TDCA, taurodeoxycholic acid; THCA, taurohyocholic acid; T $\alpha$ MCA, tauro $\alpha$-muricholic acid; T $\beta$ MCA, tauro $\beta$-muricholic 9 acid; TUDCA, tauroursodeoxycholic acid; UnconBA, unconjugated bile acid; ConBA, conjugated bile acid;PBA, primary bile 10 acid; SBA, secondary bile acid. TaurineBA,taurine conjugated bile acid; GlycineBA,glycine conjugated bile acid. 
Table 4 (on next page)

Potential biomarkers discovered by VIP value and ROC analysis 
1 Table 4 Potential biomarkers discovered by VIP value and ROC analysis

\begin{tabular}{cccc}
\hline Biomarkers & VIP & P-value & AUC \\
\hline GCDCA & 2.48 & $<0.001$ & 0.751 \\
CDCA & 2.20 & $<0.001$ & 0.815 \\
DCA & 2.06 & $<0.001$ & 0.721 \\
TCDCA & 1.41 & $<0.001$ & 0.802 \\
GCA & 1.38 & $<0.001$ & 0.774 \\
CA & 1.10 & 0.003 & 0.683 \\
\hline
\end{tabular}

2 Variable important in projection (VIP) was obtained from OPLS-DA with a threshold of 1.0; P-value was calculated from Mann-

3 Whitney U test; Area under curve (AUC) was obtained from ROC curves. 
Figure 1

Metabolomic analysis of serum samples.

(A) PCA score plots. Ellipse:Hotelling's T2 (95\%). (B) OPLS-DA score plots showing a clear discrimination between ESRD (blue sphere) and HC subjects (red octahedron).

Ellipse:Hotelling's T2 (95\%). (C) Permutation test demonstrating the robustness of OPLS-DA model. R2=0.109 and Q2=-0.198.
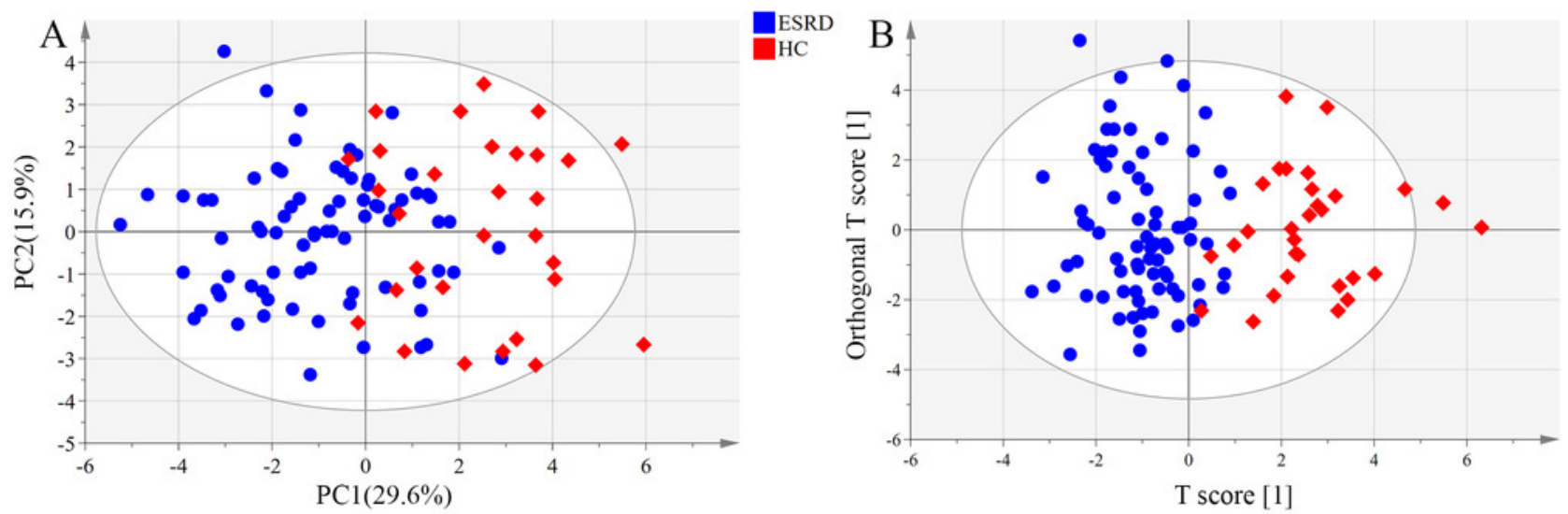

DERD

Intercepts: $\mathrm{R} 2=(0.0,0.109), \mathrm{Q} 2=(0.0,-0.198)$
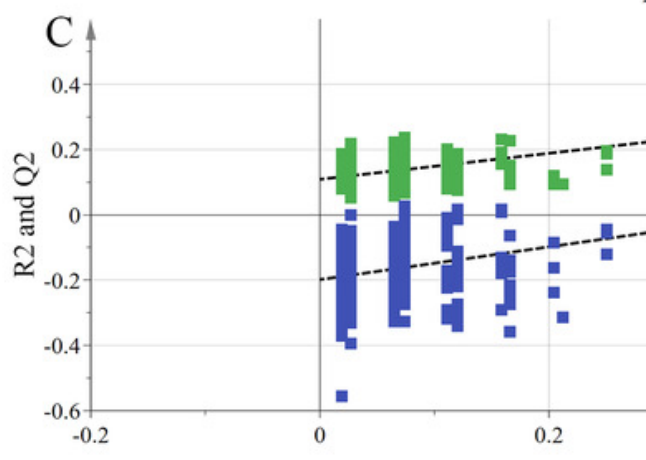
Figure 2

ROC curves for bile acid biomarkers.

ROC curves analysis for the predictive power of 6 bile acid biomarkers and TBA for distinguishing ESRD from healthy control.The final logistic model included six bile acid biomarkers. Using a cutoff probability of $50 \%$, we obtained sensitivity of $94.8 \%$ and specificity of $73.3 \%$ by ROC. The calculated area under the ROC curve was 0.890 (95\% confidence intervals, 0.818,0.963). 


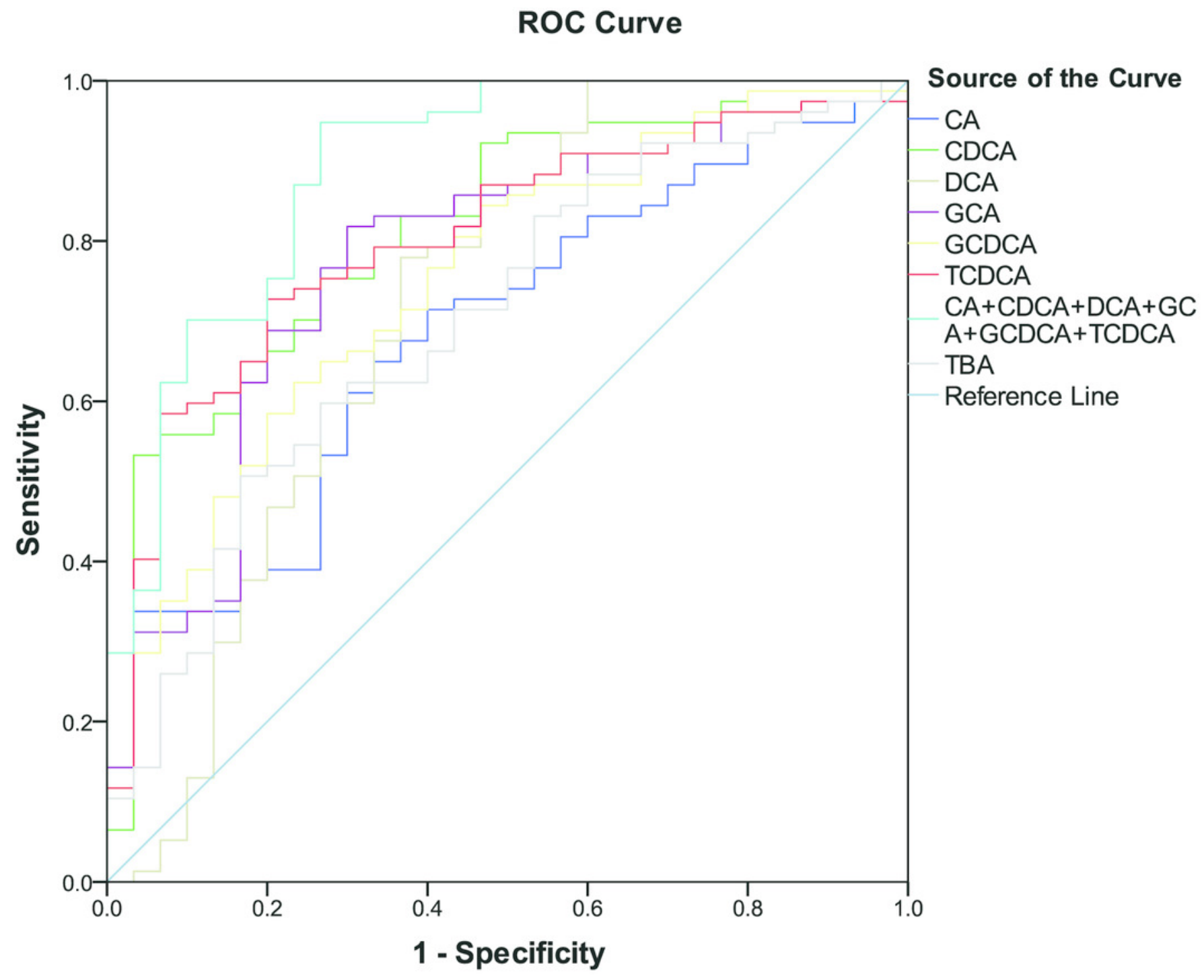




\section{Figure 3}

Differential serum bile acid profiling among the three cohorts

The fold-changes in bile acid levels between ESRD death and survival groups (relative to the control cohort) are graphically displayed. Normally-distributed data are depicted in a bar graph with means and standard errors of the mean (SEMs).*P $<0.1 ; * * P<0.05 ; * * * P<0.01$.

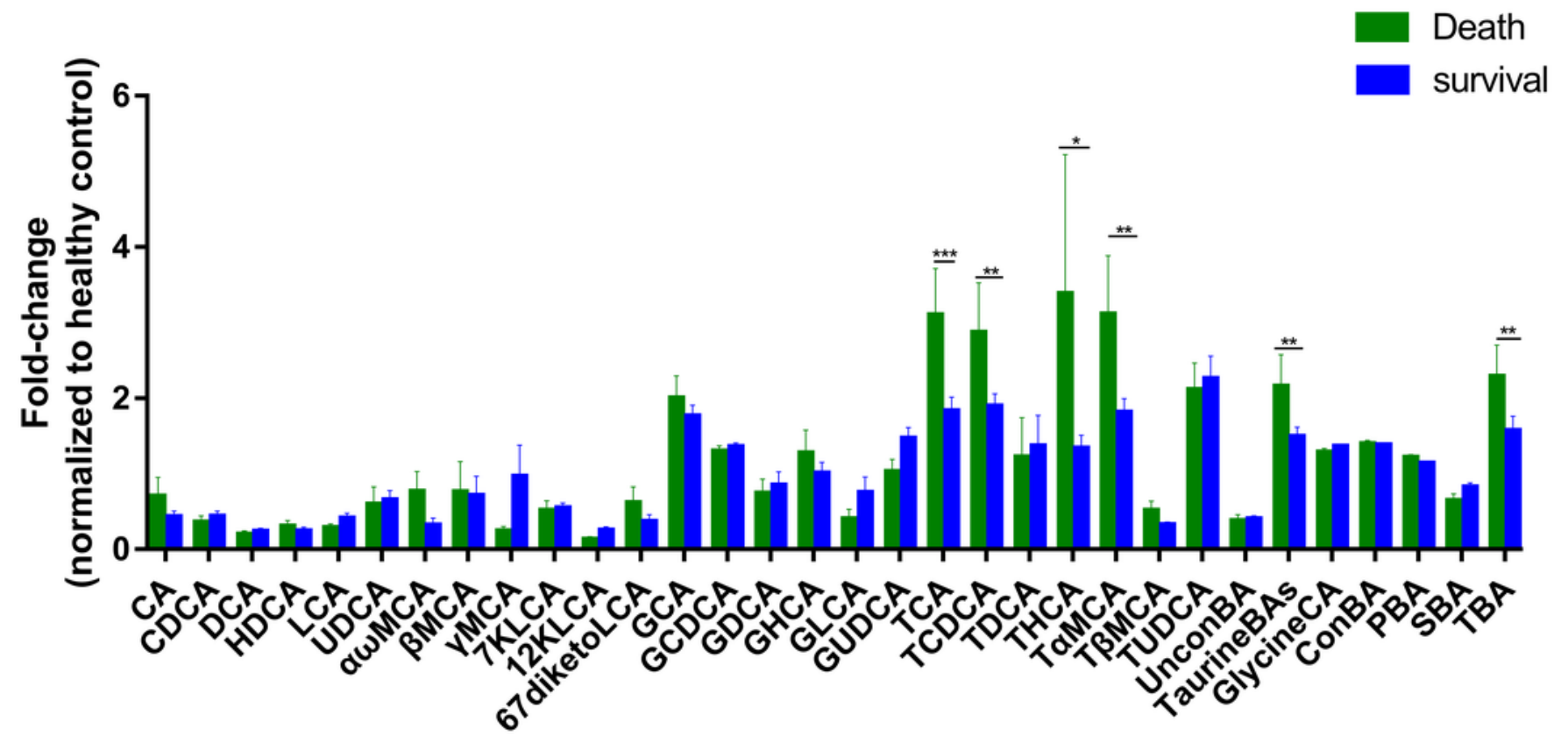

